\title{
Vibro-impact attachments as shock absorbers
}

\author{
I Karayannis ${ }^{1}$, A F Vakakis ${ }^{2,3,4 *}$, and F Georgiades ${ }^{5}$ \\ ${ }^{1}$ Department of Mechanical Engineering, National Technical University of Athens, Greece \\ ${ }^{2}$ Department of Applied Mathematical and Physical Sciences, National Technical University of Athens, Greece \\ ${ }^{3}$ Department of Mechanical and Industrial Engineering (Adjunct), University of Illinois at Urbana-Champaign, USA \\ ${ }^{4}$ Department of Aerospace Engineering (Adjunct), University of Illinois at Urbana-Champaign, USA \\ ${ }^{5}$ Aerospace and Mechanical Engineering Department, University of Liege, Belgium
}

The manuscript was received on 17 September 2007 and was accepted after revision for publication on 8 January 2008.

DOI: 10.1243/09544062JMES864

\begin{abstract}
The use of vibro-impact (VI) attachments as shock absorbers is studied. By considering different configurations of primary linear oscillators with VI attachments, the capacity of these attachments to passively absorb and dissipate significant portions of shock energy applied to the primary systems is investigated. Parametric studies are performed to determine the dependence of energy dissipation by theVI attachment in terms of its parameters. Moreover, non-linear shock spectra are used to demonstrate that appropriately designed VI attachments can significantly reduce the maximum levels of vibration of primary systems over wide frequency ranges. This is in contrast to the classical linear vibration absorber, whose action is narrowband. In addition, it is shown that VI attachments can significantly reduce or even completely eliminate resonances appearing in the linear shock spectra, thus providing strong, robust, and broadband shock protection to the primary structures to which they are attached.
\end{abstract}

Keywords: vibro-impact oscillators, shock isolation

\section{INTRODUCTION}

In recent works, interesting energy exchange phenomena in coupled oscillators have been reported, including countable infinities of co-existing branches of fundamental, subharmonic, and superharmonic periodic solutions [1] and one-way, irreversible transfer of energy from linear oscillators to non-linear attachment, termed targeted energy transfer (TET). TETs are due to transient or sustained resonance captures $[\mathbf{1}, \mathbf{2}]$, whereby the essentially non-linear attachment engages in transient resonance with the linear oscillator, before the dynamics 'escape' to different regimes of the motion $[\mathbf{3}, \mathbf{4}]$.

The regular and chaotic dynamics and bifurcations of vibro-impact (VI) oscillators have been studied extensively in the literature [5-9]. In an additional

\footnotetext{
* Corresponding author: Department of Applied Mathematical and Physical Sciences, National Technical University of Athens, PO Box 64042, GR-157 10 Zografos, Athens, Greece. email: vakakis@central.ntua.gr;avakakis@uiuc.edu
}

series of articles [10-17], VI dampers were considered to reduce the vibration levels of structures under periodic or stochastic excitation. In other works, VI devices have been applied to the problem of shock/seismic mitigation [18-20] and TET from structures to attached VI oscillators has been studied. In these words, it was shown that VI attachments are capable of absorbing broadband energy from a linear system at a fast time scale; this is critical in applications in which energy dissipation must occur at the initial phase of the motion where the energy is the highest. In the present work, the capacity of VI oscillators to passively absorb and dissipate shock energy from linear systems possessing single or more degrees-of-freedom (DOFs) is studied in detail. This is performed by parametric studies of passive energy dissipation by VI attachments and by examination of appropriately defined non-linear shock spectra. It is shown that the addition of VI oscillators with even small clearances can drastically affect the dynamics of the systems to which they are attached. This can be interpreted by the fact that VIs represent strongly non-linear, broadband perturbations of a system that 
can affect the global dynamics of a system over wide frequency ranges.

\section{SINGLE-DOF OSCILLATORS WITH VI ATTACHMENTS}

Consider the system depicted in Fig. 1 consisting of a linear single-DOF oscillator of mass $m$ coupled to an attachment with non-smooth, VI non-linearity. Apart from the weak coupling stiffness $k_{1}$, the non-linear attachment undergoes two-sided inelastic impacts when it reaches the left and right limits of the clearance $2 e$. It is assumed that the linear oscillator is forced by a half-sine shock $f(t)$ of strength $A$ and duration $T$

$$
f(t)= \begin{cases}A \sin \frac{2 \pi t}{T}, & 0 \leqslant t \leqslant \frac{T}{2} \\ 0, & t \geqslant \frac{T}{2}\end{cases}
$$

and that the integrated two-DOF system possesses zero initial conditions. The system possesses two viscous dampers with characteristics $c$ and $c_{1}$, with additional passive energy dissipation resulting from the inelastic collisions of the attachment mass $\varepsilon$. The authors model inelastic impacts by introducing the coefficient of restitution, rc, defined by the relation

$$
\mathrm{rc}=-\frac{\dot{x}_{1}^{+}-\dot{x}_{2}^{+}}{\dot{x}_{1}^{-}-\dot{x}_{2}^{-}}
$$

where the superscripts $(-)$ and $(+)$ refer to velocities before and after the impact, respectively. Clearly, the coefficient of restitution assumes values in the range $0<\mathrm{rc}<1$, with unity corresponding to perfectly elastic and zero to purely plastic impacts. Although the coefficient of restitution depends on the composition of the impacting bodies and surfaces and on the magnitudes of the velocities of the bodies during impact, in this study, it is assumed to remain constant during each individual numerical simulation.

For sufficiently large clearances, no VIs can occur, and the system becomes linear (in effect, a linear oscillation with a classical linear vibration absorber is obtained). It follows that by increasing the clearance

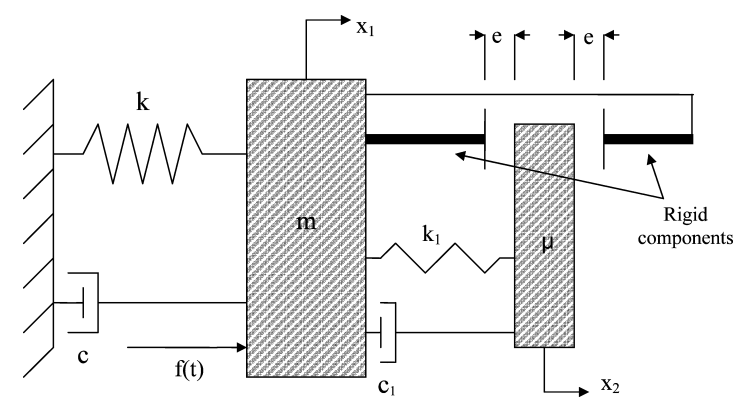

Fig. 1 Single-DOF oscillator with VI attachment to sufficiently large values, it is possible to compare the dynamics of the linear and VI systems, and, hence, assess the effects of VIs on the transient dynamics and on shock isolation.

The authors wish to study TETs from the directly forced linear oscillator to the VI attachment, leading to passive shock isolation of the linear oscillator. Eventhough the VI system under consideration is strongly non-linear, still in time intervals between impacts it behaves purely linearly. Indeed, at time windows when no VIs occur, the non-dimensionalized equations of motion of the system are given by

$$
\begin{aligned}
& \ddot{x}_{1}+\omega_{\mathrm{n}}^{2} x_{1}+\lambda \dot{x}_{1}-\lambda_{1}\left(\dot{x}_{2}-\dot{x}_{1}\right)-\Omega^{2}\left(x_{2}-x_{1}\right)=F(t) \\
& \varepsilon \ddot{x}_{2}+\Omega^{2}\left(x_{2}-x_{1}\right)+\lambda_{1}\left(\dot{x}_{2}-\dot{x}_{1}\right)=0
\end{aligned}
$$

where $\omega_{\mathrm{n}}^{2}=k / m, \Omega^{2}=k_{1} / m, \lambda=c / m, \lambda_{1}=c_{1} / m$, $\varepsilon=\mu / m$, and $F(t)=f(t) / m$.

This linear system of equations is numerically integrated until the condition of impact, $\left|x_{2}-x_{1}\right|=e$, is realized; at that time instant, continuity of displacements and discontinuity of velocities are imposed, according to the following formulas

$$
\begin{aligned}
& \dot{x}_{1}^{+}=\frac{\dot{x}_{1}^{-}(1-\varepsilon \mathrm{rc})+\varepsilon \dot{x}_{2}^{-}(\mathrm{rc}+1)}{1+\varepsilon}, \\
& \dot{x}_{2}^{+}=\frac{\dot{x}_{1}^{-}(1+\mathrm{rc})+\dot{x}_{2}^{-}(\varepsilon-\mathrm{rc})}{1+\varepsilon}
\end{aligned}
$$

and system (3a) and (b) is numerically integrated subject to the new initial conditions following the impact and until the impact condition is met again. It follows that precise computation of the time instants at which VIs occur is crucial for the correct numerical integration of the transient dynamics.

The transient dynamics of the integrated two-DOF system is simulated utilizing a Matlab code that computes precisely the time instants where impacts occur. It is emphasized that this class of VI dynamical problems is especially challenging from the analysis and from the computational point of view, as the time instants where the VIs occur are determined by the solution itself, so they cannot be determined $a$ priori, e.g. before solving the problem. Moreover, the essential (strong) non-linearity of the system is generated precisely at these time instants (due to the impulsive excitations applied to the integrated system), and in addition, at exactly these time instants, significant portions of energy are dissipated because of inelastic collisions between the attachment mass $\mu$ and the mass of the linear oscillator $m$ (due to the fact that rc $<1)$. It follows that in order to computationally model this class of problems correctly one must pay special attention to accurately compute the time instants when the VIs occur, as well as the energy dissipated 
during each impact. In addition, the accuracy of the transient computation is evaluated by checking that at each time instant of the simulation, the total initial energy of the system (provided by the external shock) is the sum of instantaneous kinetic and potential energies of the system, as well as the energy dissipated up to that time instant (by the two viscous dampers and the inelastic VIs occurred thus far).

The efficiency of the VI attachment to passively absorb and locally dissipate shock energy from the linear oscillator (which from now on will be designated as the 'primary system') is evaluated by computing an energy measure. Specifically, the portion of input shock energy that is eventually dissipated by the viscous damper with characteristic $c_{1}$ and due to the inelastic VIs is calculated. This is performed by computing the following energy dissipation measure (EDM)

$$
\begin{aligned}
& \eta_{t \gg 1}=\lim _{t \gg 1} \eta(t), \\
& \eta(t)=\frac{\int_{0}^{t} c_{1}\left[\dot{x}_{1}(\tau)-\dot{x}_{2}(\tau)\right]^{2} \mathrm{~d} \tau+\sum_{p=1}^{P} \Delta_{p}}{\int_{0}^{T} f(\tau) \dot{x}_{1}(\tau) \mathrm{d} \tau} \times 100
\end{aligned}
$$

where $\eta(t)$ is the percentage of the shock energy dissipated up to time instant $t \geqslant T ; \Delta_{p}, p=1, \ldots, P$,
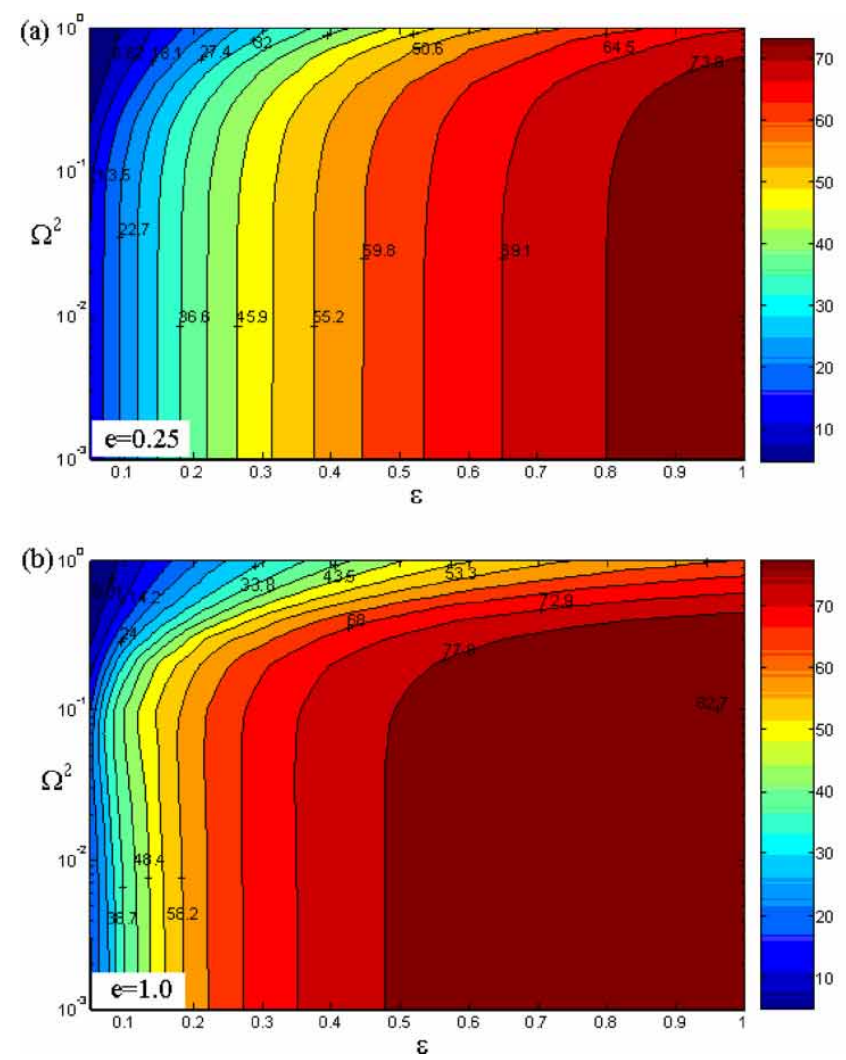

represents the amount of energy dissipated during the $p$ th VI and $P$ is the number of VIs that occur during the decaying motion up to time instant $t$. It follows that the EDM $\eta_{t \gg 1}$ is an approximation of the asymptotic limit of the time-varying measure $\eta(t)$ over the total duration of decaying motion of the system.

A series of parametric studies is performed by fixing the following parameters of the system, $m=1$, $\omega_{n}^{2}=1, \lambda=\lambda_{1}=0.1, A=10, T=0.1\left(2 \pi / \omega_{n}\right), \mathrm{rc}=0.6$, and varying the mass ratio of the VI attachment $\varepsilon$, the coupling frequency squared $\Omega^{2}$, and the clearance $e$. Figure 2 depicts the contour plots of the EDM $\eta_{t \gg 1}$ for fixed clearance and varying mass ratios and coupling frequency squared, whereas Fig. 3 presents the corresponding contour plot for the linear system (e.g. when no VIs occur for $e \approx 2.0$ ). Considering first the performance of the linear attachment (cf. Fig. 3), it is noted that the EDM reaches high values (reaching a maximum of 73.9 per cent) on a narrow zone, corresponding to the linear resonance of the attachment with the primary system. Away from this zone, the EDM deteriorates to $<50$ per cent. This is expected, as the effectiveness of the linear attachment as shock absorber is narrowband and, as a result, its performance deteriorates away from the condition of linear resonance with the primary system. In contrast, the performance of the VI attachment is broadband $[\mathbf{1 9}, \mathbf{2 0}]$, so that its
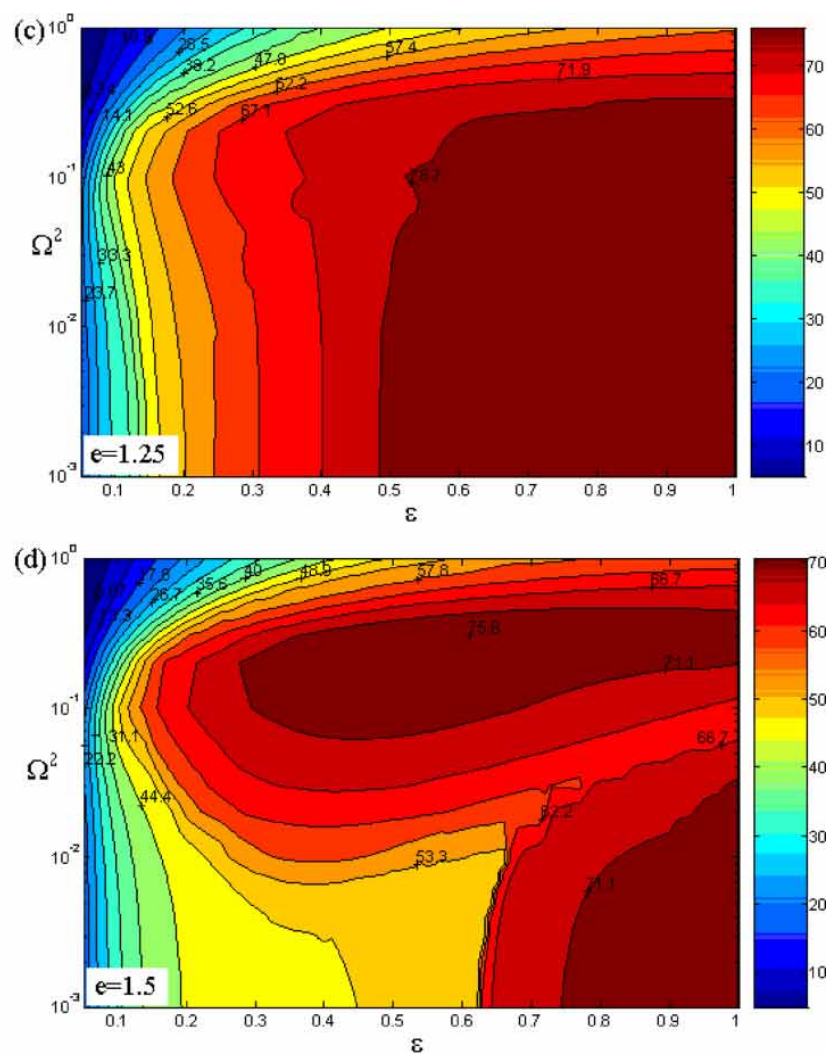

Fig. 2 Contour plots of the EDM $\eta_{t \gg 1}$ for varying $\Omega^{2}$ and $\varepsilon$ : (a) $e=0.25$, (b) $e=1.00$, (c) $e=1.25$, and (d) $e=1.50$ 


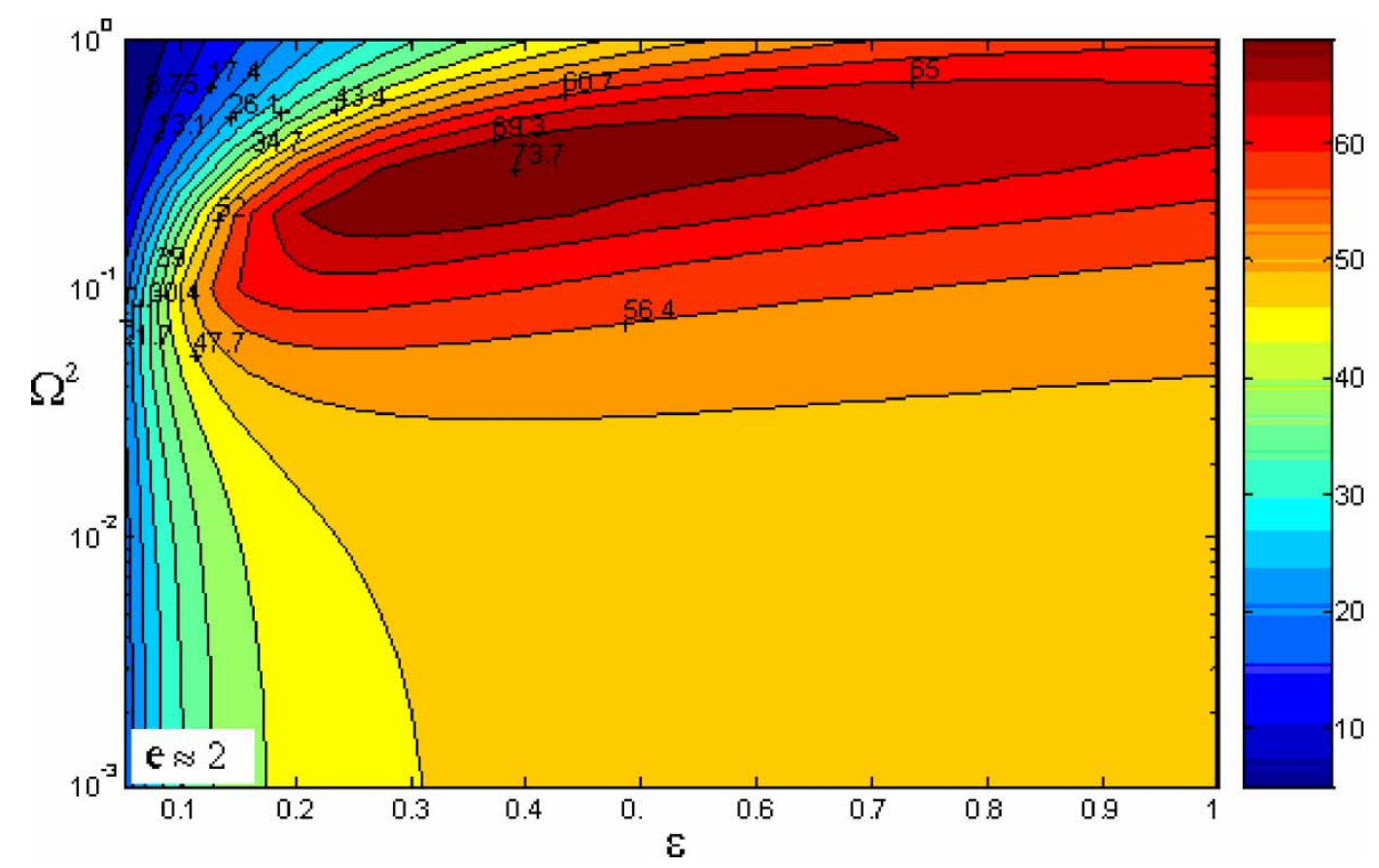

Fig. 3 Contour plots of the EDM $\eta_{t \gg 1}$ for the linear system (no VIs for $e \approx 2.0$ ) and varying $\Omega^{2}$ and $\varepsilon$

effectiveness to passively absorb and dissipate shock energy extends over wider parameter ranges.

With respect to the performance of the VI shock absorber (cf. Fig. 2), the existence of two regions in each of the EDM contour plots, corresponding to weak and stiff coupling frequencies $\Omega$ compared with the grounding frequency $\omega_{\mathrm{n}}$ of the primary system, is deduced. In the region of weak coupling frequency, an increase in the clearance of up to the value of 1.25 results in an increase in the EDM; however, further increase in the clearance results in the deterioration of the EDM values in that region of the contour plot. In contrast, in the region of stiff coupling frequency, an increase in the clearance results in an increase in the EDM and hence the effectiveness of the VI attachment. Moreover, for large values of the clearance, the EDM contour plot of the VI system tends to that of the corresponding linear plot.

Clearly, the occurrence and number of VIs affect significantly the effectiveness of vibration absorption and dissipation by the attachment. To study in more detail the dependence of the EDM on the VIs, the authors examined the differences in the EDM contour plots between the VI and linear systems and attempted to relate these differences to the number of VIs occurring in the corresponding non-linear responses. In Fig. 4, representative results that address this issue are presented; positive (negative) values of the differences in EDM correspond to surplus (deficit) of energy dissipated by the VI attachment when compared with the linear one. Clearly, the VIs play an important role in enhancing the EDM in the non-linear system. By increasing the clearance, the number of VIs diminishes, but this does not necessarily imply that the effectiveness of the VI attachment as shock isolator diminishes as well. For example, for $e=0.25$ (not shown in Fig. 4), although the number of VIs is of the order of 6000, the effectiveness of the VI attachment is not significantly enhanced compared with cases with larger clearances (cf. Fig. 3); for $e=1.50$, there occurs at most three VIs (and in most of the contour plot of Fig. 4(b), no VIs occur), yet the VI attachment attains as much as 18 per cent better EDM efficiency compared with the linear absorber in these regions. Of course, in regions where no VIs occur, the performance of the attachment converges to that of the linear absorber. It follows that efficient shock absorption efficiency from the VI attachment can be achieved even by a relatively small number of total VIs.

The previous findings can be interpreted by the fact that in the two limits $e \rightarrow 0$ and $e \approx 2$, the twoDOF system of Fig. 1 degenerates either to a linear oscillator with mass equal to $(m+\mu)$, or to a linear oscillator with a linear vibration absorber. For intermediate values of clearance and small coupling frequencies squared $\Omega^{2}$, there exist extensive regions in the parameter plane $\left(\Omega^{2}, \varepsilon\right)$ where the efficiency of the VI attachment exceeds that of the linear absorber by as much as 29 per cent, with an optimal value of clearance $e \approx 1$. In terms of absolute values of EDM, for mass ratios in the range $0.2<\varepsilon<0.5$, the EDM of 
(a)

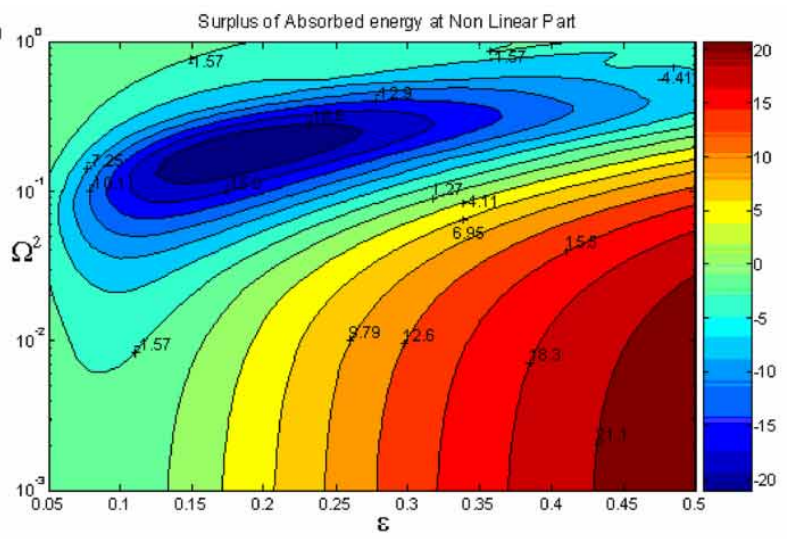

(b) $10^{\circ}$
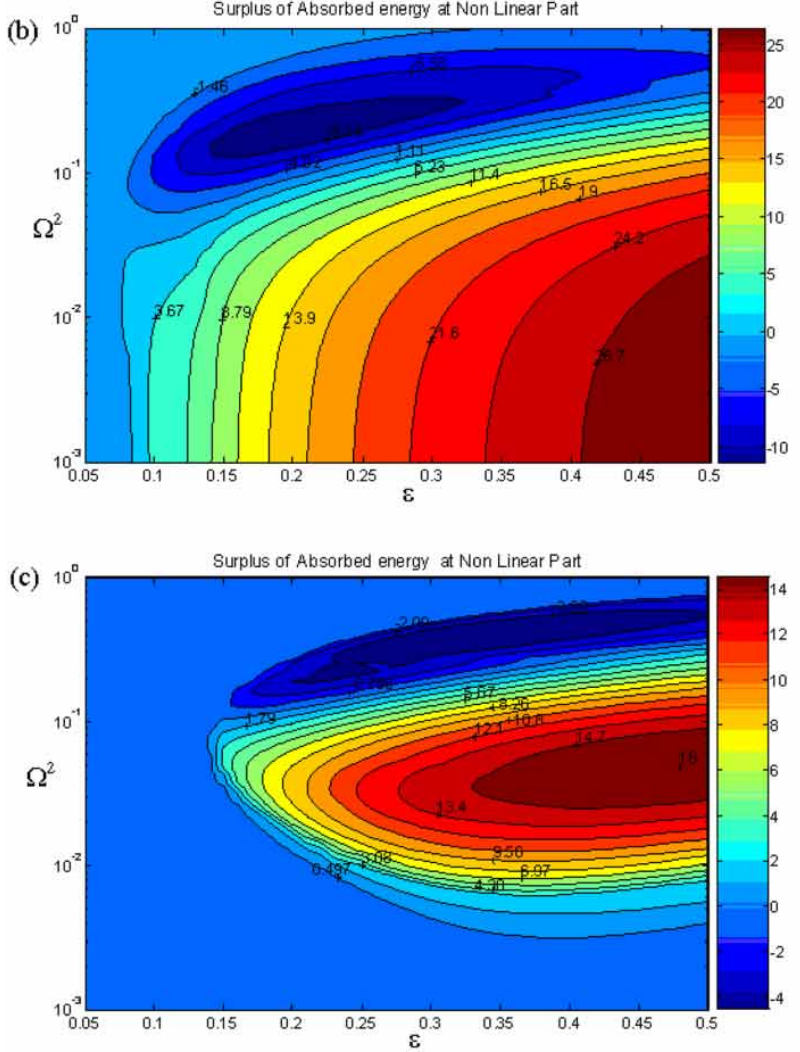
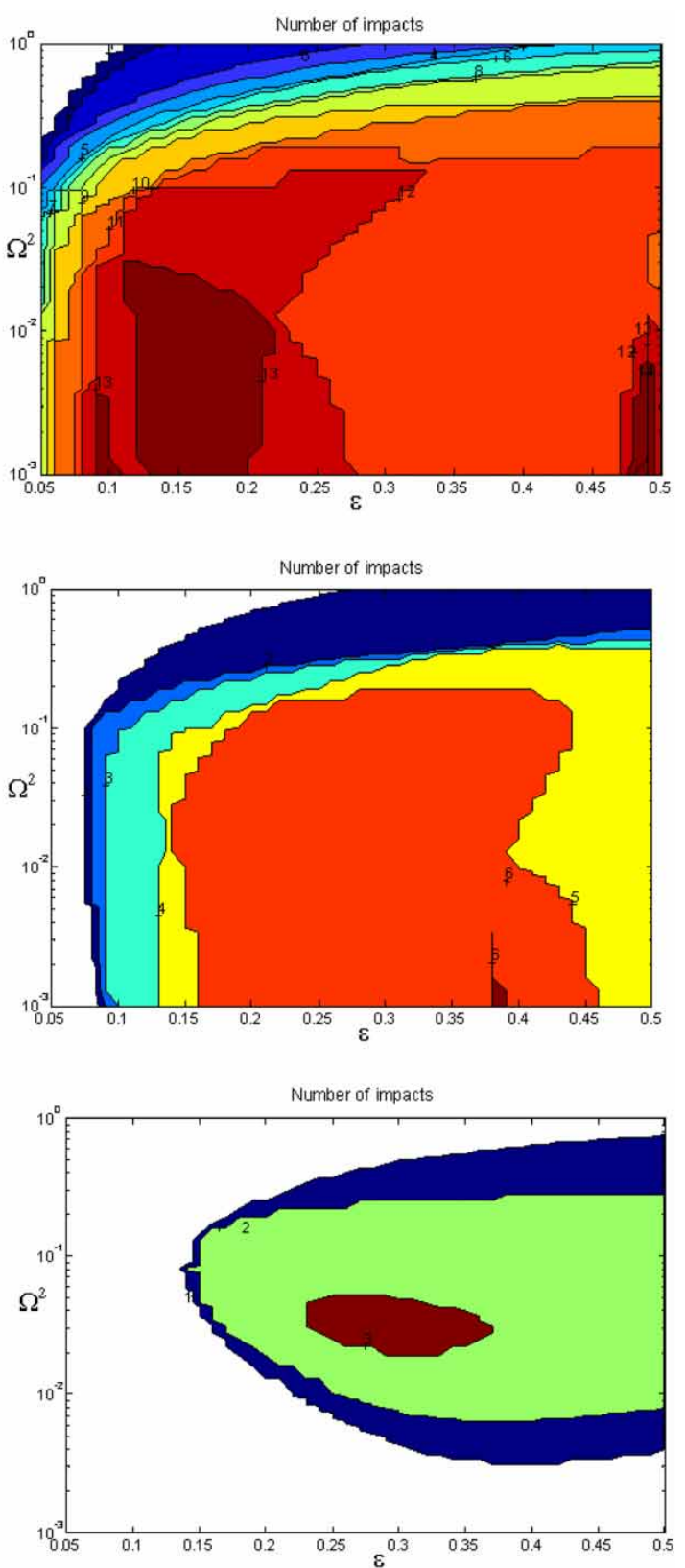

Fig. 4 Difference of EDM contour plots between the VI and linear systems and corresponding number of VI: (a) $e=0.50$, (b) $e=1.00$, (c) $e=1.50$; positive (negative) values correspond to surplus (deficit) of the energy dissipated by the VI system, compared with the linear one

the VI attachment attains values in the range 60 to 80 per cent, corresponding to a small number of total VIs (from 2 to 6).

Figure 5 depicts the plot of the EDM $\eta_{t \gg 1}$ as a function of clearance $e$ for a VI attachment with small coupling frequency $\Omega^{2}=0.005$ and mass ratio $\varepsilon=0.3$. It is noted that for this small value of coupling frequency, for small values of clearance, there is a nearly linear dependence of $\eta_{t \gg 1}$ on $e$. However, as the clearance increases to moderate values, a saturation of EDM is observed, leading to an optimal value of this measure. A further increase in $e$ results in deterioration of EDM, until above a critical threshold no VIs can occur, so the regime is linear and the EDM becomes independent of the clearance; the constant level of EDM attained above this threshold is determined by the energy dissipated by the coupling viscous damper $c_{1}$ (cf. Fig. 1). It follows that there is a definite range of clearance values, where VIs lead to enhancement of EDM, e.g. above the energy dissipated by the coupling viscous damper $c_{1}$.

From the results of Figs 3 to 5, it is noted that for small values of clearance, and in spite of the resulting relatively large total number of VIs, the shock 


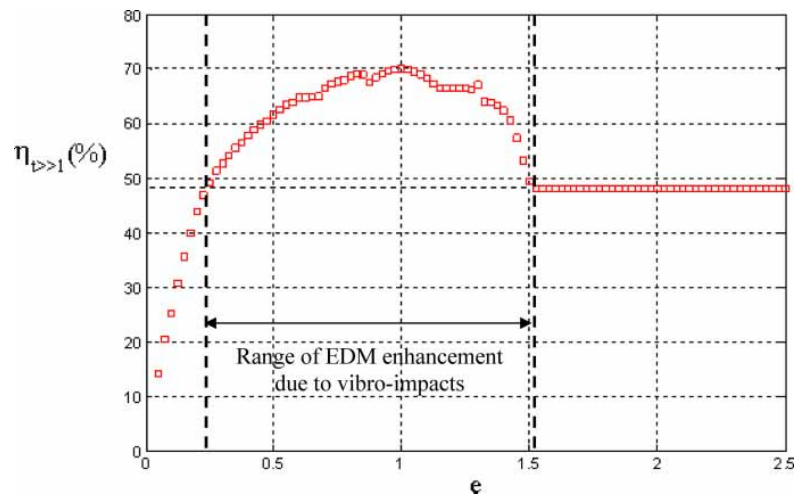

Fig. 5 EDM as a function of clearance for $\Omega^{2}=0.005$ and $\varepsilon=0.3$

absorption efficiency of the VI attachment may not be satisfactory and even inferior to that of the linear absorber. This can be inferred from the plots of Fig. 4(a) corresponding to the small clearance $e=0.5$, in which for small values of coupling frequency and mass ratio, the shock absorption efficiency of the VI attachment is comparable with that of the linear absorber, in spite of the large number of total VIs that occur at these values $(>13)$. This can be explained by noting that for small clearances and mass ratios, the relative displacements and velocities across the viscous damper $c_{1}$ are expected to be small so that small amounts of shock energy are dissipated by the damper (these results agree with the previous findings reported in reference [18]). In addition, if VIs initiate immediately after or even during the application of the external shock, for small mass ratios, the attachment is prevented from attaining sufficient acceleration and velocity, and as a result, the amount of shock energy dissipated due to VIs may be small even if a large number of VIs occur. From the plots of Fig. 4(a) it is found that by increasing the mass ratio, the EDM also increases and the regions of intensive VIs coincide with that of enhanced shock absorption efficiency.

A general conclusion from the results reported in this section is that superior shock absorption efficiency of the VI attachment compared with the linear absorber is attained for intermediate values of the clearance (e.g. away from the two limiting linear systems corresponding to zero of large clearance), small coupling frequencies, and large mass ratios. Moreover, high shock absorption efficiencies may be attained for even a small total number of VIs, provided that conditions for sufficient momentum and energy exchanges between the linear oscillator and the attachment during VIs are met.

Figure 6 shows some representative time series of the system components in order to demonstrate the effect of passive shock absorption to the response. In Figure 6(a), the responses of the linear oscillator and the linear attachment (vibration absorber) for $\Omega^{2}=$
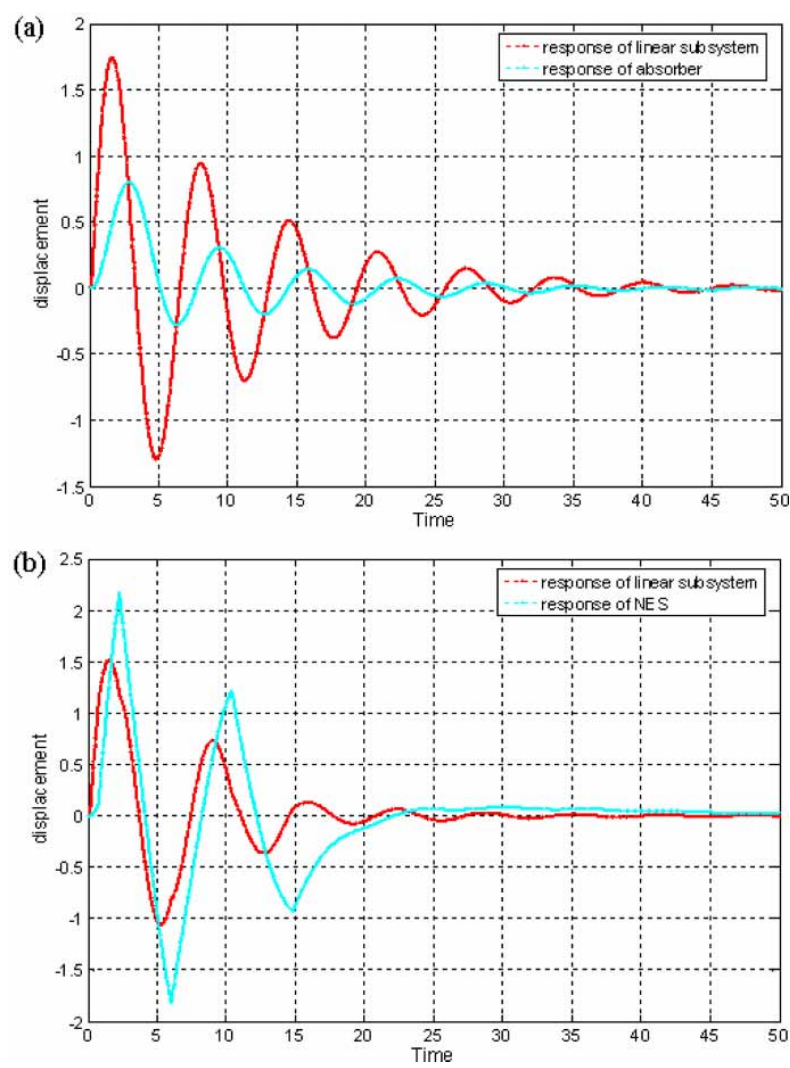

Fig. 6 Transient responses of the system with $\Omega^{2}=0.005$ and $\varepsilon=0.3:$ (a) case of linear absorber and (b) VI attachment with $e=1.0$

0.005 and $\varepsilon=0.30$, and large clearance are shown so that no VIs occur; Fig. 6(b) presents the corresponding plots for clearance $e=1.0$. From the plot of Fig. 5, it is seen that EDM attained by the VI attachment is near 70 per cent and optimal for the parameter values considered. Apart from the rapid decay of the response of the primary system due to passive shock absorption by the VI attachment, the maximum amplitude of vibration of the primary system reduces by nearly 17 per cent, compared with the corresponding value of the system with the linear absorber attached.

In the next section, the problem of reducing the maximum amplitude of vibration of a system under shock, through the use of VI attachments, is examined further. This is performed by considering non-linear shock spectra and comparing the action of the VI attachments with classical linear absorbers.

\section{SHOCK ISOLATION BY MEANS OF VI ATTACHMENTS}

Consider the system shown in Fig. 7(a) of a two-DOF linear primary system with a VI attachment. The mass $m$ represents a structure resting on an elastic foundation of mass $m_{3}$. A shock in the half-sine form (1) is 
(a)
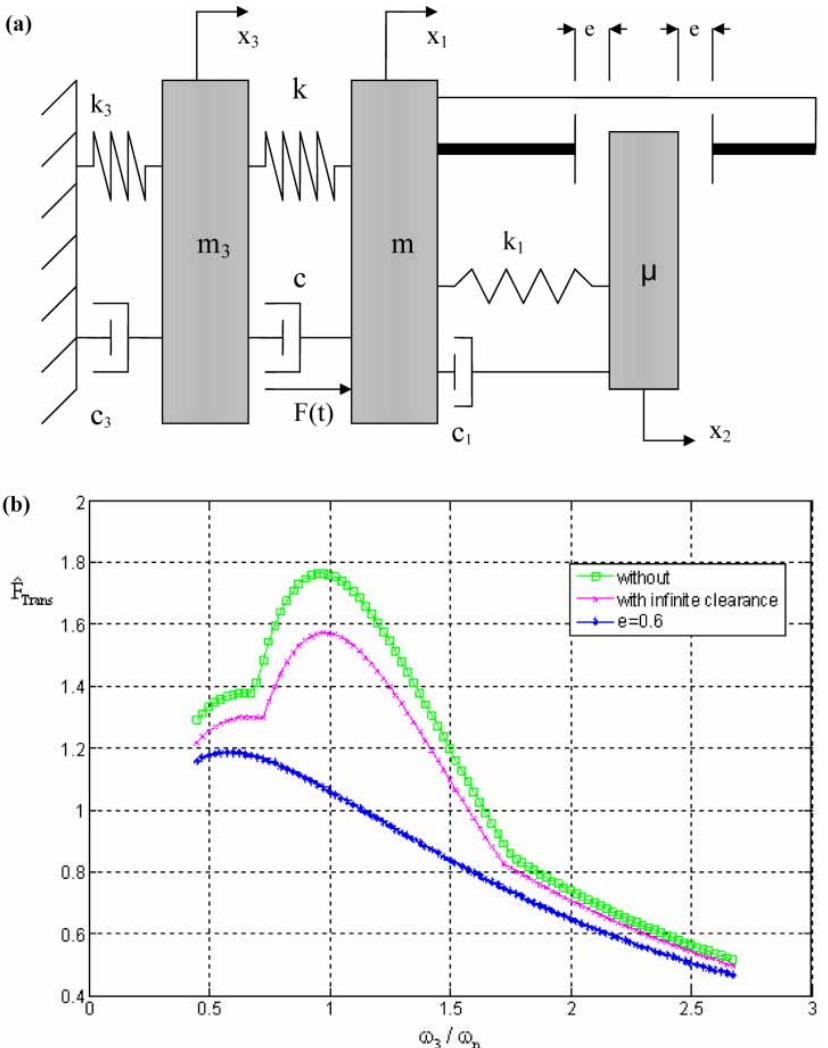

Fig. 7 Structure on foundation with VI attachment: (a) system configuration and (b) spectra of maximum normalized transmitted force to the ground as a function of non-dimensional frequency ratio

applied to the structure and the shock isolation provided by the VI attachment is studied and the results of alternative linear designs compared. The computational study is performed for the following parameter values: $m=1, m_{3} / m=5, \omega_{3}^{2}=k_{3} / m_{3}, \omega_{n}^{2}=k / m=$ $5, \lambda=c / m=0.1, \lambda_{1}=c_{1} / m=0.1, \lambda_{3}=c_{3} / m_{3}=0.1 \omega_{3}$, $e=0.6, A=10, k_{1}=0.005$, rc $=0.6, \mu=0.35$, and $T=0.2 \pi$.

Figure 7(b) shows the shock spectrum of the system with VI attachment. For comparison purposes, the non-linear spectrum is compared with the corresponding spectra of two limiting linear systems: the system obtained in the limit of infinite clearance with no VIs (e.g. the primary system attached to a linear vibration absorber) and the system obtained in the limit of zero attachment mass (e.g. the primary system with no attachment). For each plot, maximum normalized forced transmitted to the ground, $\hat{F}_{\text {Trans }}=$ $\max \left\{c_{3} \dot{x}_{3}+m_{3} \omega_{3}^{2} x_{3}\right\} /\left(J \omega_{3}\right)$, as a function of the nondimensional frequency ratio $\left(\omega_{3} / \omega_{\mathrm{n}}\right)$ is depicted. The parameter $J$ in the denominator of $\hat{F}_{\text {Trans }}$ represents the shock impulse defined by

$$
J=\int_{0}^{T} F(t) \mathrm{d} t
$$

From the results depicted in Fig. 7(b), it is inferred that the VI attachment results in significant reduction of the normalized transmitted force, which in the resonance region $\omega_{3} \approx \omega_{\mathrm{n}}$ is as much as 40 per cent compared with the case with no attachment and 32 per cent compared with the case when a linear absorber is attached. Moreover, the VI attachment eliminates the resonance response of the system and provides effective shock isolation over a wide range of frequencies. Hence, in contrast to the narrowband action of the linear vibration absorber, the VI attachment provides effective broadband shock isolation. Figure 8 shows the response $x_{3}$ of the base for the case of resonance $\omega_{3} / \omega_{\mathrm{n}}=1$ and for all three configurations discussed previously. The significant suppression of the maximum response of the system with VI attachment, as well as the suppression of the other peaks of the response and the rapid decay of the transient motion, is noted.

The second system considered is depicted in Fig. 9(a), in which the transition of shock-induced vibrations to a sensitive component (denoted by the mass $m_{3}$ ) that is connected to the mass of the primary mass $m$ is studied. A VI attachment is connected to the primary system that is also excited by the half-sine shock (1). The shock isolation study is performed for the following parameter values: $m=1$, $m_{3} / m=0.01, \omega_{3}^{2}=k_{3} / m_{3}, \omega_{\mathrm{n}}^{2}=k / m=5, \lambda=c / m=$ $0.1, \lambda_{1}=c_{1} / m=0.1, \lambda_{3}=c_{3} / m_{3}=0.1 \omega_{3}, e=0.6, A=$ $10, k_{1}=0.005$, rc $=0.6, \mu=0.35$, and $T=0.2 \pi$.

In Fig. 9, the authors depict the non-linear shock spectrum of the system with VI attachment and compare it with the two linear spectra, corresponding to systems with no and linear attachments, respectively. These spectra are computed by depicting the maximum normalized acceleration of the sensitive component $G=\max \left\{\ddot{x}_{3} /\left(\dot{u}_{m} \omega_{3}\right)\right\}$ as a function of the non-dimensional ratio $\left(\omega_{3} / \omega_{\mathrm{n}}\right)$. The velocity $\dot{u}_{\mathrm{m}}$ in

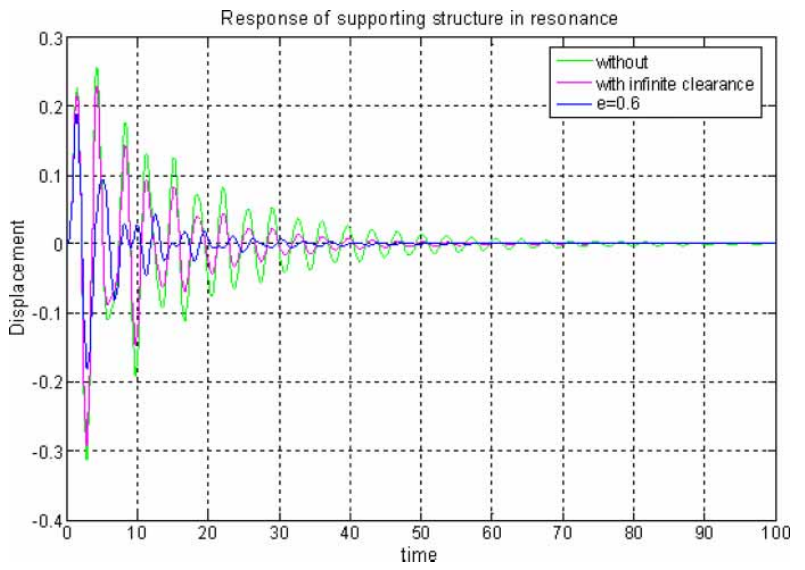

Fig. 8 Transient response $x_{3}$ of the foundation under conditions of linear resonance $\omega_{3} / \omega_{n}=1$ 
(a)

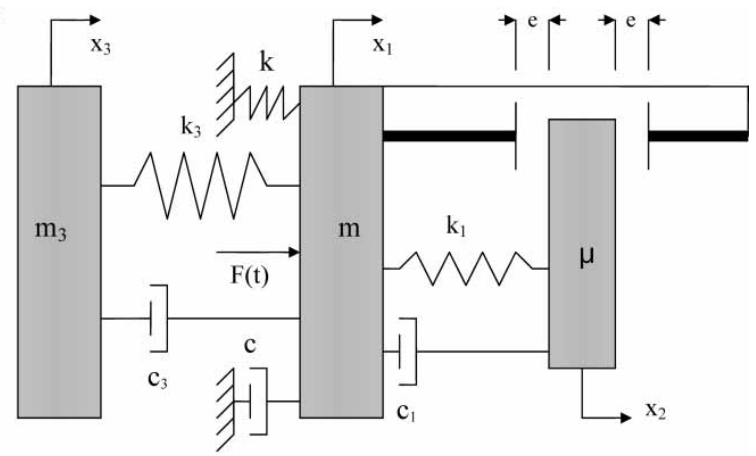

(b)

G

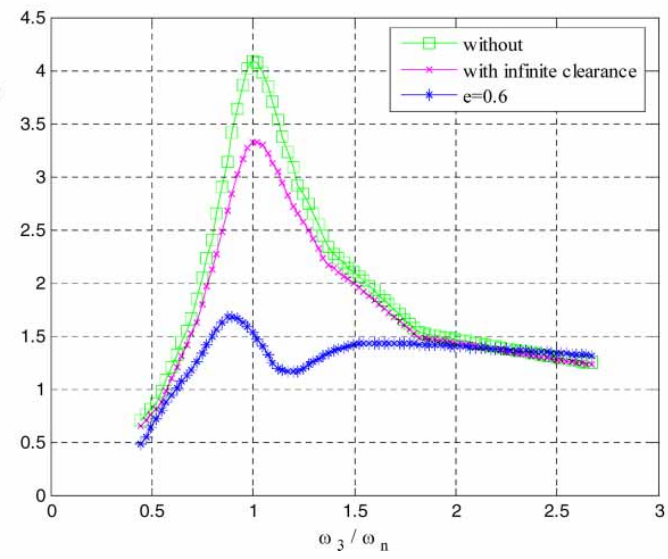

Fig.9 Structure with sensitive component and VI attachment: (a) system configuration and (b) spectra of maximum normalized acceleration of the sensitive component as a function of non-dimensional frequency ratio

the denominator of $G$ is computed by the following relation

$$
\dot{u}_{\mathrm{m}}=\frac{J}{m}, \quad J=\int_{0}^{T} F(t) \mathrm{d} t
$$

It is concluded that the VI attachment provides effective shock isolation of the sensitive component over a wide range of frequency ratios by significantly reducing the maximum normalized acceleration $G$; in the critical resonance region $\omega_{3} \approx \omega_{\mathrm{n}}$, the acceleration reduction provided by the VI attachment reaches levels of 60 per cent compared with the linear system with no attachment and 52 per cent compared with the system with linear vibration absorber attached. Moreover, as in the previous application, the VI attachment drastically reduces the resonance observed in the linear shock spectra. Figure 10 shows the displacement of the sensitive component $x_{3}$ for the three systems considered in this case under conditions of linear resonance $\omega_{3} / \omega_{n}=1$. The drastic reduction in the vibration because of the action of the VI attachment is observed.

To study the robustness of the shock isolation performance of the VI non-linear energy sink (NES),

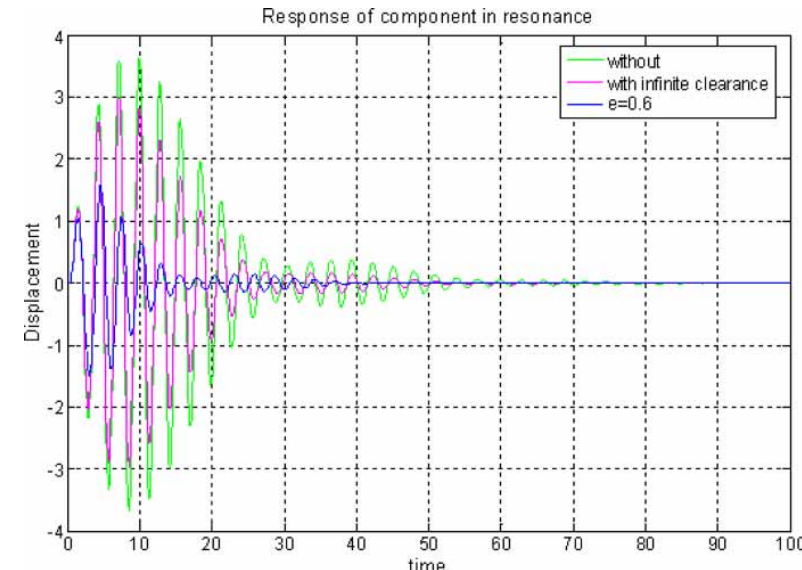

Fig. 10 Transient response $x_{3}$ of the sensitive component under conditions of linear resonance $\omega_{3} / \omega_{\mathrm{n}}=1$

an additional series of numerical simulations is performed, where for fixed impulsive excitation $(A=10$, $T=0.2 \pi$ ) and linear system parameters as defined earlier, the authors varied the parameters of the VI NES and computed shock spectra similar to that depicted in Figure 9(b); these non-linear spectra are compared with the ones of the corresponding linear systems with infinite clearance (e.g. the systems with linear absorbers attached) in order to judge the effect that VIs have on the shock isolation. In each of these plots, the system parameters that are not varied are assigned the numerical values mentioned earlier. The results are depicted in Fig. 11.

Figure 11(a) presents the comparison between the non-linear and linear spectra for five values of VI NES mass, $\mu=0.1,0.2,0.35,0.4$, and 0.5 , keeping all other NES parameters fixed. It is noted that when the mass of the VI NES increases there is better relative performance of the VI NES compared to the linear absorber; therefore, it is concluded that the mass of the VI NES is an important parameter for shock isolation (this was to be expected, since as mentioned previously, the mass of the VI NES affects the momentum exchange with the linear oscillator during VIs). Figure 11(b) shows the effect of varying the clearance $e$ on shock isolation by considering non-linear spectra for four different clearances, $e=0.1,0.2,0.4$, and 0.6 and all other NES parameters fixed. For decreasing clearance, the linear and non-linear spectra converge, with better performance of the VI NES being realized for larger clearances. From Fig. 11(c), the effect of varying the viscous damping coefficient of the VI NES is examined, considering $\lambda_{1}=0.01,0.05,0.2,0.3$, and 0.4. From these results, it is concluded that the variation of viscous damping of the VI NES does not affect significantly its effectiveness. Similarly, additional simulations (data not shown) showed that the 
(a)

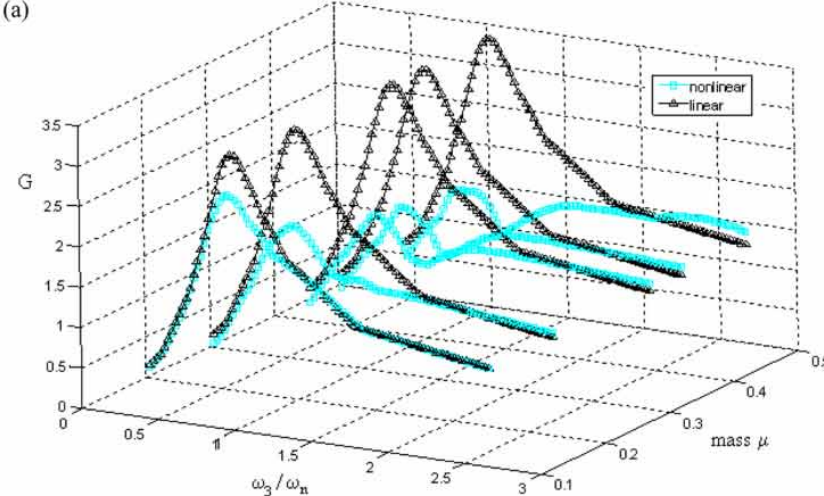

(b)

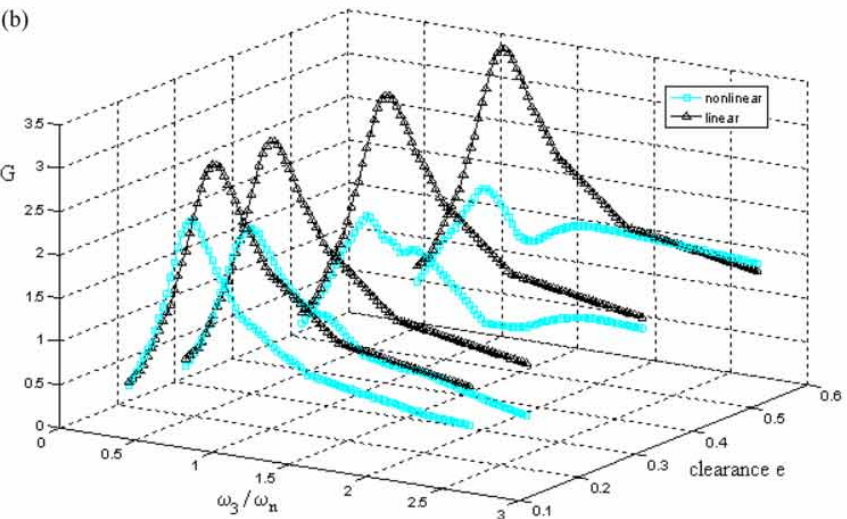

(c)

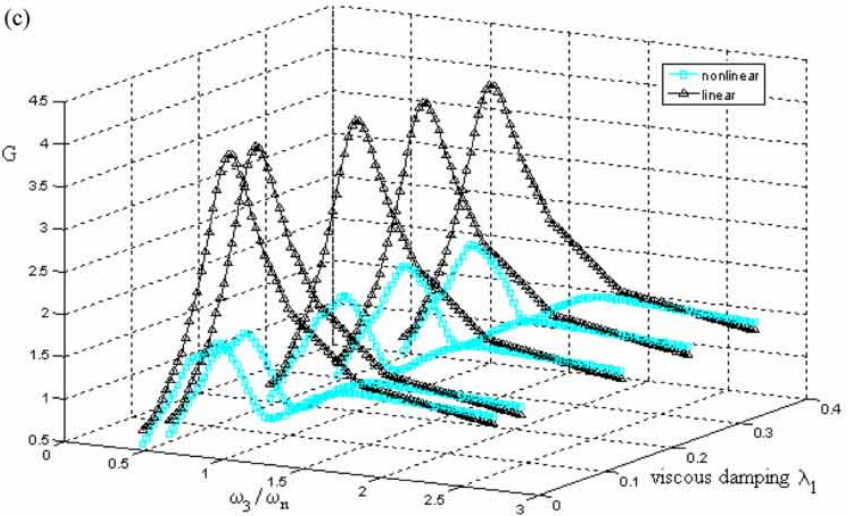

(d)

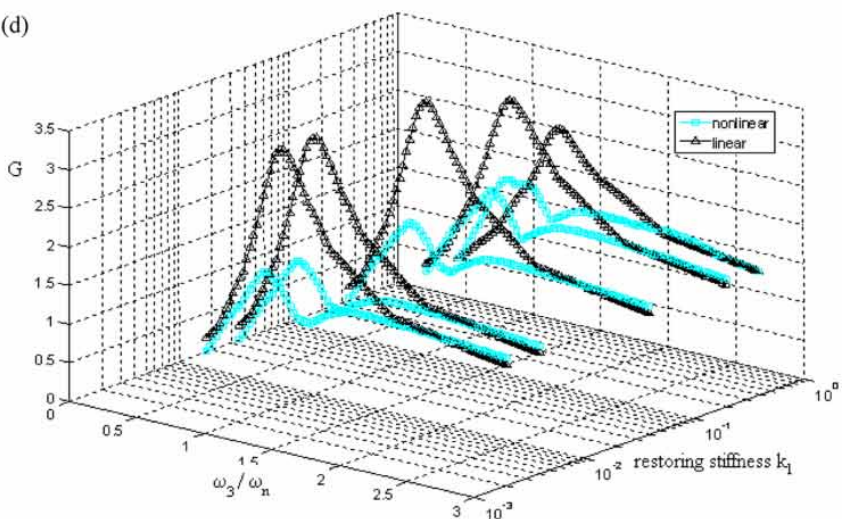

Fig. 11 Parametric study of shock spectra of maximum normalized acceleration of the sensitive component as a function of $\omega_{3} / \omega_{n}$. (a) The VI NES mass $\mu$; (b) the clearance $e$; (c) the VI NES viscous damping $\lambda_{1}$; and (d) the restoring stiffness $k_{1}$; for comparison, the corresponding linear spectra of systems with infinite clearances (e.g. with linear vibration absorbers attached) are also depicted

variation of the coefficient of restitution of the VIs from the value $\mathrm{rc}=0.6$ has a marginal effect on the performance of the VI NES. Finally, in Fig. 11(d), the effect of the restoring linear stiffness $k_{1}$ of the VI NES is studied by considering five different values: $k_{1}=5 \times 10^{-3}, 1 \times 10^{-2}, 0.1,0.5$, and 1.0. By comparing the non-linear shock spectra with the corresponding linear ones, it is noted that there is robustness of shock elimination within the examined range of stiffness values. By further increasing the stiffness $\left(k_{1}>5.0\right)$, no VIs occur and the response of the system becomes purely linear. The results presented in Fig. 11 demonstrate the robustness of the shock isolation provided by the VI NES for changes of its parameters.

\section{CONCLUDING REMARKS}

Alternative configurations of linear primary systems with VI attachments are studied. It is shown that these attachments, when properly designed, can act as effective shock isolators, providing significant reduction of maximum responses of subcomponents of the primary systems over a wide range of frequencies. Moreover, the numerical simulations considered in this work indicate that a properly designed VI attachment can provide better shock protection than the classical linear vibration absorbers of identical mass. Of course, a more complete study needs to be performed to extend this finding in a more wide range of applications.

In the design of a VI attachment as shock isolator, important design parameters are its clearance, coupling stiffness, and mass. The parametric studies performed in this work indicate that, depending on the application and the form of the applied shock, there is a range of clearances for which effective shock energy absorption and dissipation occur; outside this range, smaller or larger clearances result in the deterioration of shock absorption, as the VI attachment approaches linear limiting systems. Moreover, better energy dissipation results due to VIs are obtained for weak values of the coupling stiffness and relatively large values of mass, compared with the mass of the primary system. Of course, these conclusions concern only the system configurations studied in this work, but they are in 
agreement with findings of previous works in which VI attachments were employed in seismic mitigation designs $[\mathbf{1 9}, \mathbf{2 0}]$.

\section{ACKNOWLEDGEMENTS}

The authors wish to thank Dr Francesco Nucera of the University Reggio di Calabria, Italy, for his help with computational algorithms and codes and valuable discussions on aspects of this work.

\section{REFERENCES}

1 Lee, Y. S., Kerschen, G., Vakakis, A. F., Panagopoulos, P. N., Bergman, L. A., and McFarland, D. M. Complicated dynamics of a linear oscillator with a light, essentially nonlinear attachment. Phys. D, 2005, 204(1-2), 41-69.

2 Kerschen G., Sup Lee, Y., Vakakis, A. F., McFarland, D. M., and Bergman, L. A. Irreversible passive energy transfer in coupled oscillators with essential nonlinearity. SIAMJ. Appl. Math. 2006, 66(2), 648-679.

3 Arnold, V. I. (Ed.), Dynamical systems III, encyclopaedia of mathematical sciences 1988, vol. 3 (Springer-Verlag, Berlin and New York).

4 Quinn, D. and Rand, R. H. The dynamics of resonance capture. Nonlinear Dyn., 1995, 8, 1-20.

5 Shaw, S. W. and Rand R. H. The transition to chaos in a simple mechanical system. Int. J. Nonlinear Mech., 24, 1989, 41-56.

6 Babitsky, V. I. Theory of vibro-impact systems, 1998 (Springer-Verlag, Berlin and New York).

7 Brogliato, B. Nonsmooth mechanics, 1999 (SpringerVerlag, Berlin and New York).

8 Kryzhevich, S. G. and Pliss V. A. Chaotic modes of oscillation of a vibro-impact system. J. Appl. Math. Mech. $P M M, 2005,69,13-26$.
9 Thota, P. and Dankowicz, H. Continuous and discontinuous grazing bifurcations in impacting oscillators. Phys. $D, 2006,214,187-197$.

10 Masri, S. F. and Caughey, T. K. On the stability of the impact damper. J. Appl. Mech., 1966, 33(3), 586-592.

11 Masri, S. F. and Ibrahim A. M. Response of the impact damper to nonstationary random excitation. J. Acoust. Soc. Am., 1973, 53(1), 200-211.

12 Blazejczyk-Okolewska, B. Analysis of an impact damper of vibration. Chaos, Solitons Fractals, 2001, 12, 1983-1988.

13 Peterka, F. and Blazejczyk-Okolewska, B. Some aspects of the dynamical behavior of the impact damper. J. Vibr. Control, 2005, 11, 459-479.

14 Leine, R. I. and Nijmeijer, H. Dynamics and bifurcations in non-smooth mechanical systems, 2004 (SpringerVerlag).

15 Sun, J. Q. and Luo, A. Bifurcation and chaos in complex systems, 2006, vol. 1 (Elsevier).

16 Pfeiffer, F. and Glocker, Ch. Contacts in multibody systems. J. Appl. Math. Mech., 2000, 64(5) 773-782.

17 Filippov, A. F. Differential equations with discontinuous righthand sides, 1988 (Kluwer Academic Publishers).

18 Georgiadis, F., Vakakis, A. F., McFarland D. M., and Bergman, L. A. Shock isolation through passive energy pumping caused by non-smooth nonlinearities. Int. J. Bifur. Chaos (Special Issue on 'Non-smooth dynamical systems: recent trends and perspectives'), 2005, 15(6), $1-13$.

19 Nucera F., Vakakis, A. F., McFarland, D. M., Bergman, L. A., and Kerschen, G. Targeted energy transfer in vibro-impact oscillators for seismic mitigation. Nonlinear Dyn., 2007, 50, 651-677.

20 Nucera, F. Nonlinear energy pumping as a strategy for seismic protection. $\mathrm{PhD}$ Thesis, University of Calabria at Arcavacata of Rende - Cosenza, Italy, 2005. 